International Journal of Difference Equations (IJDE).

ISSN 0973-6069, Volume 16, Number 1, (2021). 19-34

(C) Research India Publications

https://dx.doi.org/10.37622/IJDE/16.1.2021.19-34

\title{
A Nonlocal (Two-point with Parameters) Boundary Value Problem of Differential Inclusion
}

\author{
A.M.A.EL-Sayed and W.G.EL-Sayed \\ Faculty of Science, Alexandria University, Alexandria, Egypt. \\ Somyya S.Amrajaa \\ Faculty of Science, Omar al mukhtar University, Libya
}

\begin{abstract}
Here, we study the existence of solutions of a nonlocal two-point with parameters boundary value problem of a first order nonlinear differential inclusion. The maximal and minimal solutions will be studied. The continuous dependence of the unique solution on the parameters of the nonlocal condition will be proved. The anti-periodic boundary value problem will be considered as an application
\end{abstract}

Key Words: Differential inclusion, two-point boundary value problem, existence of solutions, continuous dependence.

MSC 2010: 26A33, 34K45, 47G10.

\section{INTRODUCTION}

The models of the arbitrary (fractional-orders) differential and integral equations have many applications ( see [1], [3]-[13] and [15]-[16]). Here, we are concerning with the nonlinear differential inclusion

$$
\frac{d x}{d t} \in F_{1}\left(t, x(t), I^{\gamma} f_{2}(t, x(t))\right), \quad \gamma \in(0,1) \quad t \in(0, T)
$$

with the nonlocal two-point boundary condition

$$
\alpha x(\tau)+\beta x(\eta)=x_{0}, \quad \tau \in[0, T), \quad \eta \in(0, T], \quad \alpha, \beta>0 .
$$

We study the existence of solutions $x \in C[0, T]$ of (1)-(2). The maximal and minimal solutions and the continuous dependence of the unique solution will be study. The anti-periodic problem of (1)-(2) will be considered. 


\section{MAIN RESULTS}

The following assumptions will be needed for our goals.

(I) (i) The set $F_{1}(t, x, y)$ is nonempty, closed and convex for all $(t, x, y) \in[0, T] \in$ $R \times R$.

(ii) $F_{1}(t, x, y)$ is upper semicontinuous in $x$ and $y$ for every $t \in[0, T]$.

(iii) $F_{1}(t, x, y)$ is measurable in $t \in[0, T]$ for every $x, y \in R$.

(iv) There exists a bounded measurable function $a_{1}:[0, T] \longrightarrow R$ and a positive constant $K_{1}$, such that

$$
\left\|F_{1}(t, x, y)\right\|=\sup \left\{\left|f_{1}\right|: f_{1} \in F_{1}(t, x, y)\right\} \leq\left|a_{1}(t)\right|+K_{1}(|x|+|y|) .
$$

Remark 1 From the assumptions (i)-(iv) we can deduce that(see [1], [3]-[4] and [14]) there exists $f_{1} \in F_{1}(t, x, y)$, such that

$$
\begin{gathered}
\left|f_{1}(t, x, y)\right| \leq\left|a_{1}(t)\right|+K_{1}(|x|+|y|), \\
\frac{d x}{d t}=f_{1}\left(t, x(t), I^{\gamma} f_{2}(t, x(t))\right), \quad \gamma \in(0,1) \quad t \in(0, T) .
\end{gathered}
$$

(II) $f_{2}: I \times R \longrightarrow R$ is measurable in $t$ for any $x \in R$ and continuous in $x$ for $t \in[0, T]$ such that there exists a bounded measurable function $a_{2}(t)$ and a positive constant $K_{2}>0$ such that

$$
\left|f_{2}(t, x)\right| \leq\left|a_{2}(t)\right|+K_{2}|x|, \forall t \in I, \text { and } x \in R \text {. }
$$

(III) $\left|a_{i}(t)\right| \leq m_{i}, i=1,2$.

Remark 2 From (I) and (iv) we can deduce that every solution of (2) and (3) is a solution of (1)-(2). Now we have the following lemma.

Lemma 1.1. If the solution of the problem (2)-(3) exists then it can be expressed by the integral equation

$$
\begin{aligned}
x(t)= & \frac{1}{\alpha+\beta}\left[x_{0}-\alpha \int_{0}^{\tau} f_{1}\left(s, x(s), I^{\gamma} f_{2}(s, x(s))\right) d s\right. \\
& \left.-\beta \int_{0}^{\eta} f_{1}\left(s, x(s), I^{\gamma} f_{2}(s, x(s))\right) d s\right]+\int_{0}^{t} f_{1}\left(s, x(s), I^{\gamma} f_{2}(s, x(s))\right) d s(4)
\end{aligned}
$$

proof Let the boundary value problem (2)-(3) be satisfied. Integrating equation (3), we obtain

$$
x(t)=x(0)+\int_{0}^{t} f_{1}\left(s, x(s), I^{\gamma} f_{2}(s, x(s))\right) d s,
$$




$$
\begin{aligned}
& \alpha x(\tau)=\alpha x(0)+\alpha \int_{0}^{\tau} f_{1}\left(s, x(s), I^{\gamma} f_{2}(s, x(s))\right) d s, \\
& \beta x(\eta)=\beta x(0)+\beta \int_{0}^{\eta} f_{1}\left(s, x(s), I^{\gamma} f_{2}(s, x(s))\right) d s .
\end{aligned}
$$

Substituting equation (6) in the equation (7), we obtain

$$
x(0)=\frac{1}{\alpha+\beta}\left[x_{0}-\alpha \int_{0}^{\tau} f_{1}\left(s, x(s), I^{\gamma} f_{2}(s, x(s))\right) d s-\beta \int_{0}^{\eta} f_{1}\left(s, x(s), I^{\gamma} f_{2}(s, x(s))\right) d s\right]
$$

Substituting in (5) we get the results.

\section{EXISTENCE OF SOLUTIONS}

Theorem 2.1. Assume that the assumptions (I)-(III) are valid. Then the Problem (1)-(2) has at least one continuous solution $x \in C[0, T]$.

proof. Let $Q_{r}$ be the set $Q_{r}=\{x \in C[0, T]:\|x\| \leq r\}, t \in[0, T]$,

$$
r=\left(\frac{\left|x_{0}\right|}{\gamma+\beta}+2 m_{1} T+2 K_{1} M_{2} \frac{T^{\gamma+1}}{\Gamma(\gamma+2)}\right)\left(1-2 K_{1} T-2 K_{1} K_{2} \frac{T^{\gamma+1}}{\Gamma(\gamma+2)}\right)^{-1} .
$$

Define the operator $F$ by

$$
\begin{aligned}
& F x(t)=\frac{1}{\alpha+\beta}\left[x_{o}-\alpha \int_{0}^{\tau} f_{1}\left(s, x(s), I^{\gamma} f_{2}(s, x(s))\right) d s\right. \\
& \left.-\beta \int_{0}^{\eta} f_{1}\left(s, x(s), I^{\gamma} f_{2}(s, x(s))\right) d s\right]+\int_{0}^{t} f_{1}\left(s, x(s), I^{\gamma} f_{2}(s, x(s))\right) d s .
\end{aligned}
$$

Let $x \in Q_{r}$, then

$$
\begin{aligned}
& |F x(t)|=\mid \frac{1}{\alpha+\beta}\left[x_{0}-\alpha \int_{0}^{\tau} f_{1}\left(s, x(s), I^{\gamma} f_{2}(s, x(s))\right) d s\right. \\
& \left.-\alpha \int_{0}^{\eta} f_{1}\left(s, x(s), I^{\gamma} f_{2}(s, x(s))\right) d s\right]+\int_{0}^{t} f_{1}\left(s, x(s), I^{\gamma} f_{2}(s, x(s))\right) d s \mid \\
& \leq \frac{1}{\alpha+\beta}\left[\left|x_{0}\right|+\alpha \int_{0}^{T}\left(\left|a_{1}(s)\right|+K_{1}\left(|x(s)|+\left|I^{\gamma} f_{2}(s, x(s))\right|\right)\right) d s\right. \\
& \left.+\beta \int_{0}^{T}\left(\left|a_{1}(s)\right|+K_{1}\left(|x(s)|+\left|I^{\gamma} f_{2}(s, x(s))\right|\right)\right) d s\right] \\
& +\int_{0}^{T}\left(\left|a_{1}(s)\right|+K_{1}\left(|x(s)|+\left|I^{\gamma} f_{2}(s, x(s))\right|\right)\right) d s \\
& \leq \frac{\left|x_{0}\right|}{\alpha+\beta}+2 \int_{0}^{T}\left(\left|a_{1}(s)\right|+K_{1}\left(|x(s)|+\left|I^{\gamma} f_{2}(s, x(s))\right|\right)\right) d s \\
& +2 K_{1} K_{2}\|x\| \int_{0}^{T} \int_{0}^{s} \frac{(s-\theta)^{\gamma-1}}{\Gamma(\gamma)} d \theta d s
\end{aligned}
$$




$$
\begin{aligned}
& \leq \frac{\left|x_{0}\right|}{\alpha+\beta}+2 m_{1} T+2 K_{1} T\|x\|+2 K_{1} m_{2} \int_{0}^{T} I^{\gamma} d s+2 K_{1} K_{2}\|x\| \int_{0}^{T} \frac{s^{\gamma}}{\Gamma(\gamma+1)} d s \\
& \leq \frac{\left|x_{0}\right|}{\alpha+\beta}+2 m_{1} T+2 K_{1} T\|x\|+2 K_{1} m_{2} \int_{0}^{T} \int_{0}^{s} \frac{(s-\theta)^{\gamma-1}}{\Gamma(\gamma)} d \theta d s+2 K_{1} K_{2} r \frac{T^{\gamma+1}}{\Gamma(\gamma+2)} \\
& \leq \frac{\left|x_{0}\right|}{\alpha+\beta}+2 m_{1} T+2 K_{1} T r+2 K_{1} m_{2} \frac{T^{\gamma+1}}{\Gamma(\gamma+2)}+2 K_{1} K_{2} r \frac{T^{\gamma+1}}{\Gamma(\gamma+2)} \leq r .
\end{aligned}
$$

Thus the class of functions $\{F x\}$ is uniformly bounded on $Q_{r}$ and $F: Q_{r} \rightarrow Q_{r}$. Let $x \in Q_{r}$ and $t_{1}, t_{2} \in[0, T]$ such that $\left|t_{2}-t_{1}\right|<\delta$, then

$$
\begin{aligned}
& \left|F x\left(t_{2}\right)-F x\left(t_{1}\right)\right| \leq \int_{t_{1}}^{t_{2}}\left|f_{1}\left(s, x(s), I^{\gamma} f_{2}(s, x(s))\right)\right| d s \\
& \leq \int_{t_{1}}^{t_{2}}\left(\left|a_{1}(s)\right|+K_{1}\left(|x(s)|+I^{\gamma}\left(\left|a_{2}(s)\right|+K_{2}|x(s)|\right)\right)\right) d s \\
& \leq m_{1}\left(t_{2}-t_{1}\right)+K_{1}\left(t_{2}-t_{1}\right)\|x\| \\
& +K_{1} m_{2} \int_{t_{1}}^{t_{2}} \int_{0}^{s} \frac{(s-\theta)^{\gamma-1}}{\Gamma(\gamma)} d \theta d s+K_{1} K_{2}\|x\| \int_{t_{1}}^{t_{2}} \int_{0}^{s} \frac{(s-\theta)^{\gamma-1}}{\Gamma(\gamma)} d \theta d s \\
& \leq m_{1}\left(t_{2}-t_{1}\right)+K_{1}\left(t_{2}-t_{1}\right) r+K_{1} m_{2} \frac{t_{2}^{\gamma+1}-t_{1}^{\gamma+1}}{\Gamma(\alpha+2)}+K_{1} K_{2} r \frac{t_{2}^{\gamma+1}-t_{1}^{\gamma+1}}{\Gamma(\gamma+2)} .
\end{aligned}
$$

Thus the class of functions $\{F x\}$ is equicontinuous on $Q_{r}$ and $\{F x\}$ is compact operator by the Arzela-Ascoli Theorem[2].

Let $x_{n} \subset Q_{r}$, be convergent sequence such that, $x_{n} \rightarrow x_{0}$, then

$$
\begin{aligned}
& F x_{n}(t)=\frac{1}{\alpha+\beta}\left[x_{0}-\alpha \int_{0}^{\tau} f_{1}\left(s, x_{n}(s), I^{\gamma} f_{2}\left(s, x_{n}(s)\right)\right) d s\right. \\
& \left.-\beta \int_{0}^{\eta} f_{1}\left(s, x_{n}(s), I^{\gamma} f_{2}\left(s, x_{n}(s)\right)\right) d s\right]+\int_{0}^{t} f_{1}\left(s, x_{n}(s), I^{\gamma} f_{2}\left(s, x_{n}(s)\right)\right) d s .
\end{aligned}
$$

Using Lebesgue Dominated Convergence Theorem [2] we obtain

$$
\begin{aligned}
& \lim _{n \rightarrow \infty} F x_{n}(t)=\frac{1}{\alpha+\beta}\left[x_{0}-\alpha \lim _{n \rightarrow \infty} \int_{0}^{\tau} f_{1}\left(s, x_{n}(s), I^{\gamma} f_{2}\left(s, x_{n}(s)\right)\right) d s\right. \\
& \left.-\beta \lim _{n \rightarrow \infty} \int_{0}^{\eta} f_{1}\left(s, x_{n}(s), I^{\gamma} f_{2}\left(s, x_{n}(s)\right)\right) d s\right]+\lim _{n \rightarrow \infty} \int_{0}^{t} f_{1}\left(s, x_{n}(s), I^{\gamma} f_{2}\left(s, x_{n}(s)\right)\right) d s \\
& \leq \frac{1}{\alpha+\beta}\left[x_{0}-\alpha \int_{0}^{\tau} f_{1}\left(s, x_{0}(s), I^{\gamma} f_{2}\left(s, x_{0}(s)\right)\right) d s\right. \\
& \left.-\beta \int_{0}^{\eta} f_{1}\left(s, x_{0}(s), I^{\gamma} f_{2}\left(s, x_{0}(s)\right)\right) d s\right]+\int_{0}^{t} f_{1}\left(s, x_{0}(s), I^{\gamma} f_{2}\left(s, x_{0}(s)\right)\right) d s=F x_{0}(t) .
\end{aligned}
$$

Then $F: Q_{r} \rightarrow Q_{r}$, is continuous. Now by Schauder Fixed Point Theorem[2] there exists at least one solution $x \in C[0, T]$ of (4). 
Differentiating the integral Equation (4) we obtain

$$
\begin{aligned}
& \frac{d x}{d t}=\frac{d}{d t}\left[\frac { 1 } { \alpha + \beta } \left(x_{0}-\alpha \int_{0}^{\tau} f_{1}\left(s, x_{n}(s), I^{\gamma} f_{2}\left(s, x_{n}(s)\right)\right) d s\right.\right. \\
& \left.+\beta \int_{0}^{\eta} f_{1}\left(s, x_{n}(s), I^{\gamma} f_{2}\left(s, x_{n}(s)\right)\right) d s\right]+\int_{0}^{t} f_{1}\left(s, x_{n}(s), I^{\gamma} f_{2}\left(s, x_{n}(s)\right)\right) d s \\
= & f_{1}\left(s, x_{n}(s), I^{\gamma} f_{2}\left(s, x_{n}(s)\right)\right) \quad \text { a.e., } t \in[0, T] .
\end{aligned}
$$

Putting $t=\tau$ and multiplying equation (5) by $\alpha$, we obtain

$$
\begin{aligned}
& \alpha x(\tau)=\frac{\alpha}{\alpha+\beta} x_{0}-\frac{\alpha^{2}}{\alpha+\beta} \int_{0}^{\tau} f_{1}\left(s, x(s), I^{\gamma} f_{2}(s, x(s))\right) d s \\
& \left.-\frac{\alpha \beta}{\alpha+\beta} \int_{0}^{\eta} f_{1}\left(s, x(s), I^{\gamma} f_{2}(s, x(s))\right) d s\right]+\alpha \int_{0}^{\tau} f_{1}\left(s, x(s), I^{\gamma} f_{2}(s, x(s))\right) d s \\
& \left.=\frac{\alpha}{\alpha+\beta} x_{0}+\frac{\alpha \beta}{\alpha+\beta} \int_{0}^{\tau} f_{1}\left(s, x(s), I^{\gamma} f_{2}(s, x(s))\right) d s\right] \\
& \left.-\frac{\alpha \beta}{\alpha+\beta} \int_{0}^{\eta} f_{1}\left(s, x(s), I^{\gamma} f_{2}(s, x(s))\right) d s\right] .
\end{aligned}
$$

Also

$$
\begin{aligned}
& \left.\beta x(\tau)=\frac{\beta}{\alpha+\beta} x_{0}-\frac{\alpha \beta}{\alpha+\beta} \int_{0}^{\tau} f_{1}\left(s, x(s), I^{\gamma} f_{2}(s, x(s))\right) d s\right] \\
& \left.-\frac{\alpha \beta}{\alpha+\beta} \int_{0}^{\eta} f_{1}\left(s, x(s), I^{\gamma} f_{2}(s, x(s))\right) d s\right] .
\end{aligned}
$$

Then

$$
\alpha x(\tau)+\beta x(\eta)=x_{0}, \quad \tau \in[0, T), \quad \eta \in(0, T], \quad \alpha, \beta>0 .
$$

This prove the equivalence between the problem (2)-(3), and the integral equation(4). Then there exists at least one solution $x \in C[0, T]$ of the problem (2)-(3). Consequently, there exist at least one solution $x \in C[0, T]$ of the problem (1)-(2).

\section{MAXIMAL AND MINIMAL SOLUTIONS}

Her we study the maximal and minimal solutions for problem (1)-(2). Let $u(t)$ be a solution of (4), then $u(t)$ is said to be a maximal solution of (4) satisfies the inequality

$$
x(t) \leq u(t), \quad t \in[0, T] .
$$

A minimal solution $v(t)$ can be defined by similar way by reversing the above inequality i.e.

$$
x(t)>v(t), \quad t \in[0, T] .
$$


Lemma 3.1. Let the assumptions of Theorem 2.1 be satisfied. Assume that $x(t)$ and $y(t)$ are two continuous functions on $[0, T]$ satisfying.

$$
\begin{aligned}
& x(t) \leq \frac{1}{\alpha+\beta}\left[x_{0}-\alpha \int_{0}^{\tau} f_{1}\left(s, x(s), I^{\gamma} f_{2}(s, x(s))\right) d s\right. \\
& \left.-\beta \int_{0}^{\eta} f_{1}\left(s, x(s), I^{\gamma} f_{2}(s, x(s))\right) d s\right]+\int_{0}^{t} f_{1}\left(s, x(s), I^{\gamma} f_{2}(s, x(s))\right) d s \quad t \in[0, T] . \\
& y(t) \geq \frac{1}{\alpha+\beta}\left[x_{o}-\alpha \int_{0}^{\tau} f_{1}\left(s, y(s), I^{\gamma} f_{2}(s, y(s))\right) d s\right. \\
& \left.-\beta \int_{0}^{\eta} f_{1}\left(s, y(s), I^{\gamma} f_{2}(s, y(s))\right) d s\right]+\int_{0}^{t} f_{1}\left(s, y(s), I^{\gamma} f_{2}(s, y(s))\right) d s \quad t \in[0, T]
\end{aligned}
$$

where one of them is strict. Let functions $f_{1}$ and $f_{2}$ are monotonic nondecreasing in $x$, then

$$
x(t)<y(t), \quad t>0
$$

Proof. Let the conclusion (8) not true, then there exists $t_{1}$ with

$$
x\left(t_{1}\right)<y\left(t_{1}\right), \quad t_{1}>0 \quad \text { and } x(t)<y(t), \quad 0<t<t_{1} .
$$

For $f_{1}$ and $f_{2}$ are monotonic functions in $x$ we have

$$
\begin{aligned}
& x\left(t_{1}\right) \leq \frac{1}{\alpha+\beta}\left[x_{o}-\alpha \int_{0}^{\tau} f_{1}\left(s, x(s), I^{\gamma} f_{2}(s, x(s))\right) d s\right. \\
& \left.-\beta \int_{0}^{\eta} f_{1}\left(s, x(s), I^{\gamma} f_{2}(s, x(s))\right) d s\right]+\int_{0}^{t_{1}} f_{1}\left(s, x(s), I^{\gamma} f_{2}(s, x(s))\right) d s . \\
& <\frac{1}{\alpha+\beta}\left[x_{o}-\alpha \int_{0}^{\tau} f_{1}\left(s, y(s), I^{\gamma} f_{2}(s, y(s))\right) d s\right. \\
& \left.-\beta \int_{0}^{\eta} f_{1}\left(s, y(s), I^{\gamma} f_{2}(s, y(s))\right) d s\right]+\int_{0}^{t_{1}} f_{1}\left(s, y(s), I^{\gamma} f_{2}(s, y(s))\right) d s<y\left(t_{1}\right) .
\end{aligned}
$$

This contrasts with the fact that $x\left(t_{1}\right)=y\left(t_{1}\right)$. This complete the proof. For the existence of the continuous maximal and minimal solutions for (4), we have the following theorem.

Theorem 3.2. . Let assumptions of Theorem 2.1, be hold. Moreover, if $f_{1}$ and $f_{2}$ are monotonic nondecreasing functions in $x$ for each $t \in[0, T]$, then the equation (4) has maximal and minimal solutions.

proof. First, we must demonstrate the existence of the maximal solution of (4). Let 
$\epsilon>0$ be given. Now consider the integral equation

$$
\begin{aligned}
x_{\epsilon_{1}}(t) & =\frac{1}{\alpha+\beta}\left[x_{0}-\alpha \int_{0}^{\tau} f_{1}\left(s, x_{\epsilon_{1}}(s), I^{\gamma} f_{2}\left(s, x_{\epsilon_{1}}(s)\right)\right) d s\right. \\
& \left.-\beta \int_{0}^{\eta} f_{1}\left(s, x_{\epsilon_{1}}(s), I^{\gamma} f_{2}\left(s, x_{\epsilon_{1}}(s)\right)\right) d s\right] \\
& +\int_{0}^{t} f_{1}\left(s, x_{\epsilon_{1}}(s), I^{\gamma} f_{2}\left(s, x_{\epsilon_{1}}(s)\right)\right) d s \quad t \in[0, T]
\end{aligned}
$$

where $f_{2}\left(s, x_{\epsilon}(s)\right)=f_{2}\left(s, x_{\epsilon}(s)\right)+\epsilon$

$$
\begin{gathered}
x_{\epsilon_{2}}(t)=\frac{1}{\alpha+\beta}\left[x_{0}-\alpha \int_{0}^{\tau} f_{1}\left(s, x_{\epsilon_{2}}(s), I^{\gamma} f_{2}\left(s, x_{\epsilon_{2}}(s)\right)\right) d s\right. \\
\left.-\beta \int_{0}^{\eta} f_{1}\left(s, x_{\epsilon_{2}}(s), I^{\gamma} f_{2}\left(s, x_{\epsilon_{2}}(s)\right)\right) d s\right]+\int_{0}^{t} f_{1}\left(s, x_{\epsilon_{2}}(s), I^{\gamma} f_{2}\left(s, x_{\epsilon_{2}}(s)\right)\right) d s . \\
x_{\epsilon_{2}}(t)=\frac{1}{\alpha+\beta}\left[x_{0}-\alpha \int_{0}^{\tau}\left(f_{1}\left(s, x_{\epsilon_{2}}(s), I^{\gamma} f_{2}\left(s, x_{\epsilon_{2}}(s)\right)\right)+\epsilon_{2}\right) d s\right. \\
\left.-\beta \int_{0}^{\eta}\left(f_{1}\left(s, x_{\epsilon_{2}}(s), I^{\gamma} f_{2}\left(s, x_{\epsilon_{2}}(s)\right)\right)+\epsilon_{2}\right) d s\right] \\
+\int_{0}^{t}\left(f_{1}\left(s, x_{\epsilon_{2}}(s), I^{\gamma} f_{2}\left(s, x_{\epsilon_{2}}(s)\right)\right)+\epsilon_{2}\right) d s
\end{gathered}
$$

$$
\begin{aligned}
x_{\epsilon_{1}}(t)= & \frac{1}{\alpha+\beta}\left[x_{0}-\alpha \int_{0}^{\tau} f_{1}\left(s, x_{\epsilon_{1}}(s), I^{\gamma} f_{2}\left(s, x_{\epsilon_{1}}(s)\right)\right) d s\right. \\
& \left.-\beta \int_{0}^{\eta} f_{1}\left(s, x_{\epsilon_{1}}(s), I^{\gamma} f_{2}\left(s, x_{\epsilon_{1}}(s)\right)\right) d s\right]+\int_{0}^{t} f_{1}\left(s, x_{\epsilon_{1}}(s), I^{\gamma} f_{2}\left(s, x_{\epsilon_{1}}(s)\right)\right) d s . \\
& x_{\epsilon_{1}}(t)=\frac{1}{\alpha+\beta}\left[x_{0}-\alpha \int_{0}^{\tau}\left(f_{1}\left(s, x_{\epsilon_{1}}(s), I^{\alpha} f_{2}\left(s, x_{\epsilon_{1}}(s)\right)\right)+\epsilon_{1}\right) d s\right. \\
& \left.-\beta \int_{0}^{\eta}\left(f_{1}\left(s, x_{\epsilon_{1}}(s), I^{\gamma} f_{2}\left(s, x_{\epsilon_{1}}(s)\right)\right)+\epsilon_{1}\right) d s\right] \\
& +\int_{0}^{t}\left(f_{1}\left(s, x_{\epsilon_{1}}(s), I^{\gamma} f_{2}\left(s, x_{\epsilon_{1}}(s)\right)\right)+\epsilon_{1}\right) d s . \\
& >\frac{1}{\alpha+\beta}\left[x_{0}-\alpha \int_{0}^{\tau}\left(f_{1}\left(s, x_{\epsilon_{2}}(s), I^{\gamma} f_{2}\left(s, x_{\epsilon_{2}}(s)\right)\right)+\epsilon_{2}\right) d s\right. \\
& \left.-\beta \int_{0}^{\eta}\left(f_{1}\left(s, x_{\epsilon_{2}}(s), I^{\alpha} f_{2}\left(s, x_{\epsilon_{2}}(s)\right)\right)+\epsilon_{2}\right) d s\right] \\
& +\int_{0}^{t}\left(f_{1}\left(s, x_{\epsilon_{2}}(s), I^{\gamma} f_{2}\left(s, x_{\epsilon_{2}}(s)\right)\right)+\epsilon_{2}\right) d s .
\end{aligned}
$$

Applying Lemma 2, we obtain $x_{\epsilon_{2}}<x_{\epsilon_{1}}, t \in[0, T]$.

As shown before the family of function $x_{\epsilon}(t)$ is equi-continuous and uniformly 
bounded, then by Arzela Theorem, there exist decreasing sequence $\epsilon_{n}$, such that $\epsilon_{0} \rightarrow 0$ as $n \rightarrow \infty$, and $u(t)=\lim _{n \rightarrow \infty} x_{\epsilon_{n}}(t)$ exists uniformly in $[0, T]$ and denote this limit by $u(t)$. From the continuity of the functions, $f_{2 \epsilon}\left(t, x_{\epsilon}(t)\right)$, we get $f_{2 \epsilon}\left(t, x_{\epsilon}(t)\right) \longrightarrow f_{2}(t, x(t))$ as $n \rightarrow \infty$ and

$$
\begin{aligned}
& u(t)=\lim _{n \rightarrow \infty} x_{\epsilon_{n}}(t)=\frac{1}{\alpha+\beta}\left[x_{0}-\alpha \int_{0}^{\tau} f_{1}\left(s, x(s), I^{\alpha} f_{2}(s, x(s))\right) d s\right. \\
& \left.-\beta \int_{0}^{\eta} f_{1}\left(s, x(s), I^{\alpha} f_{2}(s, x(s))\right) d s\right]+\int_{0}^{t} f_{1}\left(s, x(s), I^{\alpha} f_{2}(s, x(s))\right) d s .
\end{aligned}
$$

Now we prove that $u(t)$ is the maximal solution of (4). To do this, let $x(t)$ be any solution of (4), then

$$
\begin{gathered}
x(t)=\frac{1}{\alpha+\beta}\left[x_{0}-\alpha \int_{0}^{\tau} f_{1}\left(s, x(s), I^{\gamma} f_{2}(s, x(s))\right) d s\right. \\
\left.-\beta \int_{0}^{\eta} f_{1}\left(s, x(s), I^{\gamma} f_{2}(s, x(s))\right) d s\right]+\int_{0}^{t} f_{1}\left(s, x(s), I^{\gamma} f_{2}(s, x(s))\right) d s, \\
x_{\epsilon}(t)=\frac{1}{\alpha+\beta}\left[x_{0}-\alpha \int_{0}^{\tau} f_{1}\left(s, x_{\epsilon}(s), I^{\gamma} f_{2}\left(s, x_{\epsilon}(s)\right)\right) d s\right. \\
\left.-\beta \int_{0}^{\eta} f_{1}\left(s, x_{\epsilon}(s), I^{\gamma} f_{2}\left(s, x_{\epsilon}(s)\right)\right) d s\right]+\int_{0}^{t} f_{1}\left(s, x_{\epsilon}(s), I^{\gamma} f_{2}\left(s, x_{\epsilon}(s)\right)\right) d s . \\
x_{\epsilon}(t)=\frac{1}{\alpha+\beta}\left[x_{0}-\alpha \int_{0}^{\tau}\left(f_{1}\left(s, x_{\epsilon}(s), I^{\gamma} f_{2}\left(s, x_{\epsilon}(s)\right)\right)+\epsilon\right) d s\right. \\
\left.-\beta \int_{0}^{\eta}\left(f_{1}\left(s, x_{\epsilon}(s), I^{\gamma} f_{2}\left(s, x_{\epsilon}(s)\right)\right)+\epsilon\right) d s\right]+\int_{0}^{t} f_{1}\left(\left(s, x_{\epsilon}(s), I^{\gamma} f_{2}\left(s, x_{\epsilon}(s)\right)\right)+\epsilon\right) d s . \\
>\frac{1}{\alpha+\beta}\left[x_{o}-\alpha \int_{0}^{\tau} f_{1}\left(s, x_{\epsilon}(s), I^{\gamma} f_{2}\left(s, x_{\epsilon}(s)\right)\right) d s\right. \\
\left.-\beta \int_{0}^{\eta} f_{1}\left(s, x_{\epsilon}(s), I^{\alpha} f_{2}\left(s, x_{\epsilon}(s)\right)\right) d s\right]+\int_{0}^{t} f_{1}\left(s, x_{\epsilon}(s), I^{\gamma} f_{2}\left(s, x_{\epsilon}(s)\right)\right) d s=x(t) .
\end{gathered}
$$

Applying lemma (3.1), we obtain $x(t)<x_{\epsilon}(t), t \in[0, T]$.

From the uniqueness of the maximal solution it clear that $x_{\epsilon}(t)$ tends to $u(t)$ uniformly in $[0, T]$ as $\epsilon \rightarrow 0$ by similar way as done above we can prove the existence of the minimal solution.

\section{UNIQUENESS OF THE SOLUTION}

Here we study the sufficient conditions for the uniqueness of the solution $x \in C[0, T]$, of problems (1)-(2). Consider the following assumptions 
(I) (i) The set $F_{1}(t, x, y)$ is nonempty, compact and convex for all $(t, x, y) \in[0, T] \in$ $R \times R$.

(ii) $F_{1}$ is continuous and satisfies Lipschitz condition with a positive constant $K_{1}$ such that

$$
H\left(F_{1}\left(t, x_{1}, y_{1}\right), F_{1}\left(t, x_{2}, y_{2}\right) \mid \leq K_{1}\left(\left|x_{1}-x_{2}\right|+\left|y_{1}-y_{2}\right|\right)\right.
$$

where $H(A, B)$ is the Hausdorff metric between the two subsets $A, B \in I \times E$ (see[13])

Remark 3 From this assumptions we can deduce that there exists a function $f_{1} \in F_{1}(t, x, y)$, such that

(iii) $f_{1}: I \times R \times R \rightarrow R$ is continuous and satisfies Lipschitz condition with a positive constant $K_{1}$ such that (see [2],[3] and [14])

$$
\begin{gathered}
\left|f_{1}\left(t, x_{1}, y_{1}\right)-f_{1}\left(t, x_{2}, y_{2}\right)\right| \leq K_{1}\left(\left|x_{1}-x_{2}\right|+\left|y_{1}-y_{2}\right|\right), \\
\frac{d x}{d t}=f_{1}\left(t, x(t), I^{\gamma} f_{2}(t, x(t))\right), \quad \gamma \in(0,1) \quad t \in(0, T) .
\end{gathered}
$$

(II) $f_{2}: I \times R \longrightarrow R$, is continuous and satisfies Lipschitz condition with positive constant $K_{2}$, such that

$$
\left|f_{2}(t, x)-f_{2}(t, y)\right| \leq K_{2}|x-y|
$$

From this assumption (I), we have

$$
\left|f_{1}(t, x, y)\right|-\left|f_{1}(t, 0,0)\right| \leq\left|f_{1}(t, x, y)-f_{1}(t, 0,0)\right| \leq K_{1}(|x|+|y|) .
$$

Then

$$
\left|f_{1}(t, x, y)\right| \leq K_{1}(|x|+|y|)+\left|f_{1}(t, 0,0)\right| \leq K_{1}(|x|+|y|)+\left|a_{1}(t)\right|
$$

where $\left|a_{1}(t)\right|=\sup _{t \in I}\left|f_{1}(t, 0,0)\right|$ and from assumption (II), we have

$$
\left|f_{2}(t, x)\right|-\left|f_{2}(t, 0)\right| \leq\left|f_{2}(t, x)-f_{2}(t, 0)\right| \leq K_{2}|x| .
$$

Then

$$
\left|f_{2}(t, x)\right| \leq K_{2}|x|+\left|f_{2}(t, 0)\right| \leq K_{2}|x|+\left|a_{2}(t)\right|, \quad\left|a_{2}(t)\right|=\sup _{t \in I}\left|f_{1}(t, 0)\right| .
$$

Theorem 4.1. Let the assumptions (I)-(II) be satisfies. Then the solution of the Problem (2)-(3) is a unique. 
Proof Let $x_{1}(t)$ and $x_{2}(t)$ be solution of the problem (2)-(3), then

$$
\begin{aligned}
& \left|x_{1}(t)-x_{2}(t)\right|=\mid \frac{1}{\alpha+\beta}\left[x_{0}-\alpha \int_{0}^{\tau} f_{1}\left(s, x_{1}(s), I^{\gamma} f_{2}\left(s, x_{1}(s)\right)\right) d s\right. \\
& \left.-\beta \int_{0}^{\eta} f_{1}\left(s, x_{1}(s), I^{\gamma} f_{2}\left(s, x_{1}(s)\right)\right) d s\right]+\int_{0}^{t} f_{1}\left(s, x_{1}(s), I^{\gamma} f_{2}\left(s, x_{1}(s)\right)\right) d s \\
& -\left(\frac { 1 } { \alpha + \beta } \left[x_{0}-\alpha \int_{0}^{\tau} f_{1}\left(s, x_{2}(s), I^{\gamma} f_{2}\left(s, x_{2}(s)\right)\right) d s\right.\right. \\
& \left.\left.-\beta \int_{0}^{\eta} f_{1}\left(s, x_{2}(s), I^{\gamma} f_{2}\left(s, x_{2}(s)\right)\right) d s\right]+\int_{0}^{t} f_{2}\left(s, x_{2}(s), I^{\gamma} f_{2}\left(s, x_{2}(s)\right)\right) d s\right) \mid \\
& \leq \frac{\alpha}{\alpha+\beta} \int_{0}^{\tau}\left|f_{1}\left(s, x_{2}(s), I^{\gamma} f_{2}\left(s, x_{2}(s)\right)\right)-f_{1}\left(s, x_{1}(s), I^{\gamma} f_{2}\left(s, x_{1}(s)\right)\right)\right| d s \\
& +\frac{\beta}{\alpha+\beta} \int_{0}^{\eta}\left|f_{1}\left(s, x_{2}(s), I^{\gamma} f_{2}\left(s, x_{2}(s)\right)\right)-f_{1}\left(s, x_{1}(s), I^{\gamma} f_{2}\left(s, x_{1}(s)\right)\right)\right| d s \\
& +\int_{0}^{t}\left|f_{1}\left(s, x_{1}(s), I^{\gamma} f_{2}\left(s, x_{1}(s)\right)\right)-f_{1}\left(s, x_{2}(s), I^{\gamma} f_{2}\left(s, x_{2}(s)\right)\right)\right| d s \\
& \leq 2 \int_{0}^{T}\left|f_{1}\left(s, x_{1}(s), I^{\gamma} f_{2}\left(s, x_{1}(s)\right)\right)-f_{1}\left(s, x_{2}(s), I^{\gamma} f_{2}\left(s, x_{2}(s)\right)\right)\right| d s \\
& \leq 2 K_{1} \int_{0}^{T}\left(\left|x_{2}(s)-x_{1}(s)\right|+\left|I^{\gamma} f_{2}\left(s, x_{2}(s)\right)-I^{\gamma} f_{2}\left(s, x_{1}(s)\right)\right|\right) d s \\
& \leq 2 K_{1}\left\|x_{1}-x_{2}\right\| T+2 K_{1} \int_{0}^{T} I^{\gamma}\left|f_{2}\left(s, x_{2}(s)\right)-f_{2}\left(s, x_{1}(s)\right)\right| d s \\
& \leq 2 K_{1}\left\|x_{1}-x_{2}\right\| T+2 K_{1} K_{2}\left\|x_{1}-x_{2}\right\| \int_{0}^{T} \frac{s^{\gamma}}{\Gamma(\gamma+1)} d s \\
& \leq 2 K_{1}\left\|x_{1}-x_{2}\right\| T+2 K_{1} K_{2} \int_{0}^{\gamma}\left|x_{2}(s)-x_{1}(s)\right| d s \\
& \leq x_{1}-x_{2}\left\|T+2 K_{1} K_{2}\right\| x_{1}-x_{2} \| \int_{0}^{T} \frac{(s-\theta)^{\gamma-1}}{\Gamma(\gamma)} d \theta d s \\
& \leq K_{1} K_{2} \frac{T^{\gamma+1}}{\Gamma(\gamma+2)}\left\|x_{1}-x_{2}\right\| \\
& \leq \\
& \leq
\end{aligned}
$$

Hence $\left(1-\left(T+K_{2} \frac{T^{\gamma+1}}{\Gamma(\gamma+2)}\right) 2 K_{1}\right)<1$, then $x_{1}(t)=x_{2}(t)$.

The solution of (2)-(3) is unique. This completes the proof.

\section{CONTINUOUS DEPENDENCE OF THE SOLUTION}

Definition 1. The unique solution of the problem (1)-(2) depends continuously on 
initial data $x_{0}$, if $\epsilon>0, \exists \delta>0$ such that

$$
\left|x_{0}-x_{0}^{*}\right| \leq \delta \quad \Rightarrow\left\|x-x^{*}\right\| \leq \epsilon
$$

where $x^{*}$ is the unique solution of

$$
\begin{aligned}
& x^{*}(t)=\frac{1}{\alpha+\beta}\left[x_{0}^{*}-\alpha \int_{0}^{\tau} f_{1}\left(s, x^{*}(s), I^{\gamma} f_{2}\left(s, x^{*}(s)\right)\right) d s\right. \\
& \left.-\beta \int_{0}^{\eta} f_{1}\left(s, x^{*}(s), I^{\gamma} f_{2}\left(s, x^{*}(s)\right)\right) d s\right]+\int_{0}^{t} f_{1}\left(s, x^{*}(s), I^{\gamma} f_{2}\left(s, x^{*}(s)\right)\right) d s
\end{aligned}
$$

Theorem 5.1. Let the assumption of Theorem 2.1 be satisfied, then the unique solution of (1)-(2) depends continuously on $x_{0}$

Proof. Let $x(t)$ and $x^{*}(t)$ be the solutions of problem (1)-(2), then

$$
\begin{aligned}
& \left|x(t)-x^{*}(t)\right|=\mid \frac{1}{\alpha+\beta}\left[x_{0}-\alpha \int_{0}^{\tau} f_{1}\left(s, x(s), I^{\gamma} f_{2}(s, x(s))\right) d s\right. \\
& \left.-\beta \int_{0}^{\eta} f_{1}\left(s, x(s), I^{\gamma} f_{2}(s, x(s))\right) d s\right]+\int_{0}^{t} f_{1}\left(s, x(s), I^{\gamma} f_{2}(s, x(s))\right) d s \\
& -\left(\frac { 1 } { \alpha + \beta } \left[x_{0}^{*}-\alpha \int_{0}^{\tau} f_{1}\left(s, x^{*}(s), I^{\gamma} f_{2}\left(s, x^{*}(s)\right)\right) d s\right.\right. \\
& \left.\left.-\beta \int_{0}^{\eta} f_{1}\left(s, x^{*}(s), I^{\gamma} f_{2}\left(s, x^{*}(s)\right)\right) d s\right]+\int_{0}^{t} f_{1}\left(s, x^{*}(s), I^{\gamma} f_{2}\left(s, x^{*}(s)\right)\right) d s\right) \mid \\
& \leq \frac{1}{\alpha+\beta}\left|x_{0}-x_{0}^{*}\right| \\
& +\frac{\alpha}{\alpha+\beta} \int_{0}^{\tau} \mid f_{1}\left(s, x(s), I^{\gamma} f_{2}(s, x(s))-f_{1}\left(s, x^{*}(s), I^{\gamma} f_{2}\left(s, x^{*}(s)\right) \mid d s\right.\right. \\
& +\frac{\beta}{\alpha+\beta} \int_{0}^{\eta} \mid f_{1}\left(s, x(s), I^{\gamma} f_{2}(s, x(s))-f_{1}\left(s, x^{*}(s), I^{\gamma} f_{2}\left(s, x^{*}(s)\right) \mid d s\right.\right. \\
& +\int_{0}^{t} \mid f_{1}\left(s, x(s), I^{\gamma} f_{2}(s, x(s))-f_{1}\left(s, x^{*}(s), I^{\gamma} f_{2}\left(s, x^{*}(s)\right) \mid d s\right.\right. \\
& \leq \frac{1}{\alpha+\beta}\left|x_{0}-x_{0}^{*}\right|+2 \int_{0}^{T} \mid f_{1}\left(s, x(s), I^{\gamma} f_{2}(s, x(s))-f_{1}\left(s, x^{*}(s), I^{\gamma} f_{2}\left(s, x^{*}(s)\right) \mid d s\right.\right. \\
& \leq \frac{1}{\alpha+\beta}\left|x_{0}-x_{0}^{*}\right|+2 \int_{0}^{T}\left(K_{1}\left|x(s)-x^{*}(s)\right|+K_{1}\left|I^{\gamma} f_{2}(s, x(s))-I^{\gamma} f_{2}\left(s, x^{*}(s)\right)\right|\right) d s \\
& \leq \frac{1}{\alpha+\beta}\left|x_{0}-x_{0}^{*}\right|+2 K_{1}\left\|x-x^{*}\right\| T+2 K_{1} K_{2} \frac{T^{\gamma+1}}{\Gamma(\gamma+2)}\left\|x-x^{*}\right\| \\
& \leq \frac{\delta}{\alpha+\beta}+\left(2 K_{1} T+2 K_{1} K_{2} \frac{T^{\gamma+1}}{\Gamma(\gamma+2)}\right)\left\|x-x^{*}\right\| . \\
&
\end{aligned}
$$

Hence

$$
\left\|x-x^{*}\right\| \leq \frac{\delta}{\alpha+\beta}\left(1-\left(2 K_{1} T+2 K_{1} K_{2} \frac{T^{\gamma+1}}{\Gamma(\alpha+2)}\right)\right)^{-1}=\epsilon .
$$


Definition 2. The unique solution of (1)-(2) depends continuously on initial $\alpha$ and $\beta$ if $\epsilon>0, \exists \delta>0$, such that

$$
\left|\alpha-\alpha^{*}\right| \leq \delta,\left|\beta-\beta^{*}\right| \leq \delta \quad \Rightarrow\left\|x-x^{*}\right\| \leq \epsilon
$$

Theorem 5.2. Let the assumption of Theorem 2.1 be satisfied, then the solution of (1)-(2), depends continuously on $\alpha$ and $\beta$.

Proof. Let $x(t)$ and $x^{*}(t)$ be the solutions of problem (1)-(2), then

$$
\begin{aligned}
& \left|x(t)-x^{*}(t)\right|=\mid \frac{x_{0}}{\alpha+\beta}-\frac{\alpha}{\alpha+\beta} \int_{0}^{\tau} f_{1}\left(s, x(s), I^{\gamma} f_{2}(s, x(s))\right) d s \\
& \left.-\frac{\beta}{\alpha+\beta} \int_{0}^{\eta} f_{1}\left(s, x(s), I^{\gamma} f_{2}(s, x(s))\right) d s\right]+\int_{0}^{t} f_{1}\left(s, x(s), I^{\gamma} f_{2}(s, x(s))\right) d s \\
& -\frac{x_{0}}{\alpha_{*}+\beta_{*}}+\frac{\alpha^{*}}{\alpha_{*}+\beta_{*}} \int_{0}^{\tau} f_{1}\left(s, x^{*}(s), I^{\gamma} f_{2}\left(s, x^{*}(s)\right)\right) d s \\
& +\frac{\beta^{*}}{\alpha_{*}+\beta_{*}} \int_{0}^{\eta} f_{1}\left(s, x^{*}(s), I^{\gamma} f_{2}\left(s, x^{*}(s)\right)\right) d s-\int_{0}^{t} f_{1}\left(s, x^{*}(s), I^{\gamma} f_{2}\left(s, x^{*}(s)\right)\right) d s \mid \\
& \leq\left|\frac{x_{0}}{(\alpha+\beta)}-\frac{x_{0}}{\left(\alpha^{*}+\beta^{*}\right)}\right| \\
& +\left|\frac{\alpha^{*}}{\left(\alpha^{*}+\beta^{*}\right)} \int_{0}^{\tau} f_{1}\left(s, x^{*}(s), I^{\gamma} f_{2}\left(s, x^{*}(s)\right)\right) d s-\frac{\alpha}{\alpha+\beta} \int_{0}^{\tau} f_{1}\left(s, x(s), I^{\gamma} f_{2}(s, x(s))\right) d s\right| \\
& +\left|\frac{\beta^{*}}{\left(\alpha^{*}+\beta^{*}\right)} \int_{0}^{\eta} f_{1}\left(s, x^{*}(s), I^{\gamma} f_{2}\left(s, x^{*}(s)\right)\right) d s-\frac{\beta}{\alpha+\beta} \int_{0}^{\eta} f_{1}\left(s, x(s), I^{\gamma} f_{2}(s, x(s))\right) d s\right| \\
& +\int_{0}^{t}\left|f_{1}\left(s, x^{*}(s), I^{\gamma} f_{2}\left(s, x^{*}(s)\right)\right)-f_{1}\left(s, x(s), I^{\gamma} f_{2}(s, x(s))\right)\right| d s \\
& \leq \frac{\left|x_{0}\right|\left(\left|\alpha-\alpha_{*}\right|+\left|\beta-\beta^{*}\right|\right)}{(\alpha+\beta)\left(\alpha^{*}+\beta^{*}\right)} \\
& +\left|\frac{\alpha^{*}}{\left(\alpha^{*}+\beta^{*}\right)} \int_{0}^{\tau}\right| f_{1}\left(s, x^{*}(s), I^{\gamma} f_{2}\left(s, x^{*}(s)\right)\right)\left|d s-\frac{\alpha}{\alpha+\beta} \int_{0}^{\tau} f_{1}\left(s, x^{*}(s), I^{\gamma} f_{2}\left(s, x^{*}(s)\right)\right) d s\right| \\
& +\left|\frac{\alpha}{\alpha+\beta} \int_{0}^{\tau} f_{1}\left(s, x^{*}(s), I^{\gamma} f_{2}\left(s, x^{*}(s)\right)\right) d s-\frac{\alpha}{\alpha+\beta} \int_{0}^{\tau} f_{1}\left(s, x(s), I^{\gamma} f_{2}(s, x(s))\right) d s\right| \\
& +\left|\frac{\beta^{*}}{\left(\alpha^{*}+\beta^{*}\right)} \int_{0}^{\eta} f_{1}\left(s, x^{*}(s), I^{\gamma} f_{2}\left(s, x^{*}(s)\right)\right) d s-\frac{\beta}{\alpha+\beta} \int_{0}^{\eta} f_{1}\left(s, x^{*}(s), I^{\gamma} f_{2}\left(s, x^{*}(s)\right)\right) d s\right| \\
& +\left|\frac{\beta}{\alpha+\beta} \int_{0}^{\eta} f_{1}\left(s, x^{*}(s), I^{\gamma} f_{2}\left(s, x^{*}(s)\right)\right) d s-\frac{\beta}{\alpha+\beta} \int_{0}^{\eta} f_{1}\left(s, x(s), I^{\gamma} f_{2}(s, x(s))\right) d s\right| \\
& +\int_{0}^{t}\left|f_{1}\left(s, x^{*}(s), I^{\gamma} f_{2}\left(s, x^{*}(s)\right)\right)-f_{1}\left(s, x(s), I^{\gamma} f_{2}(s, x(s))\right)\right| d s \\
& \leq \frac{\left|x_{0}\right|\left(\left|\alpha-\alpha_{*}\right|+\left|\beta-\beta^{*}\right|\right)}{(\alpha+\beta)\left(\alpha^{*}+\beta^{*}\right)} \\
& +\left|\frac{\alpha^{*}}{\left(\alpha^{*}+\beta^{*}\right)}-\frac{\alpha}{(\alpha+\beta)}\right| \int_{0}^{\tau}\left|f_{1}\left(s, x^{*}(s), I^{\gamma} f_{2}\left(s, x^{*}(s)\right)\right)\right| d s
\end{aligned}
$$




$$
\begin{aligned}
& +\frac{\alpha}{\alpha+\beta} \int_{0}^{\tau}\left|f_{1}\left(s, x^{*}(s), I^{\gamma} f_{2}\left(s, x^{*}(s)\right)\right)-f_{1}\left(s, x(s), I^{\gamma} f_{2}(s, x(s))\right)\right| d s \\
& +\left|\frac{\beta^{*}}{\left(\alpha^{*}+\beta^{*}\right)}-\frac{\beta}{(\alpha+\beta)}\right| \int_{0}^{\tau}\left|f_{1}\left(s, x^{*}(s), I^{\gamma} f_{2}\left(s, x^{*}(s)\right)\right)\right| d s \\
& +\frac{\beta}{\alpha+\beta} \int_{0}^{\eta}\left|f_{1}\left(s, x^{*}(s), I^{\gamma} f_{2}\left(s, x^{*}(s)\right)\right)-f_{1}\left(s, x(s), I^{\gamma} f_{2}(s, x(s))\right)\right| d s \\
& +\int_{0}^{t}\left|f_{1}\left(s, x^{*}(s), I^{\gamma} f_{2}\left(s, x^{*}(s)\right)\right)-f_{1}\left(s, x(s), I^{\gamma} f_{2}(s, x(s))\right)\right| d s \\
& \leq \frac{\left|x_{0}\right|(\delta+\delta)}{(\alpha+\beta)\left(\alpha^{*}+\beta^{*}\right)}+\left[\left|\frac{\alpha^{*}}{\left(\alpha^{*}+\beta^{*}\right)}-\frac{\alpha}{\alpha+\beta}\right|\right. \\
& \left.+\left|\frac{\beta^{*}}{\left(\alpha^{*}+\beta^{*}\right)}-\frac{\beta}{\alpha+\beta}\right|\right] \int_{0}^{T} f_{1}\left(s, x^{*}(s), I^{\gamma} f_{2}\left(s, x^{*}(s)\right)\right) d s \\
& +2 \int_{0}^{T}\left|f_{1}\left(s, x^{*}(s), I^{\gamma} f_{2}\left(s, x^{*}(s)\right)\right)-f_{1}\left(s, x(s), I^{\gamma} f_{2}(s, x(s))\right)\right| d s \\
& \leq \frac{(2 \delta)\left|x_{0}\right|}{(\alpha+\beta)\left(\alpha^{*}+\beta^{*}\right)} \\
& +\left(\frac{\left|\alpha^{*} \beta-\alpha \beta^{*}\right|}{(\alpha+\beta)\left(\alpha^{*}+\beta^{*}\right)}+\frac{\left|\alpha^{*} \beta-\alpha \beta^{*}\right|}{(\alpha+\beta)\left(\alpha^{*}+\beta^{*}\right)}\right) \int_{0}^{T} f_{1}\left(s, x^{*}(s), I^{\gamma} f_{2}\left(s, x^{*}(s)\right)\right) d s \\
& +\left(2 K_{1} T+2 K_{1} K_{2} \frac{T^{\gamma+1}}{\Gamma(\gamma+2)}\right)\left\|x-x^{*}\right\| \leq \frac{(2 \delta)\left|x_{0}\right|}{(\alpha+\beta)\left(\alpha^{*}+\beta^{*}\right)} \\
& +\left[\frac{\left|\alpha^{*} \beta-\alpha \beta\right|+\left|\alpha \beta-\alpha \beta^{*}\right|}{(\alpha+\beta)\left(\alpha^{*}+\beta^{*}\right)}\right. \\
& \left.\left.+\frac{\left|\alpha^{*} \beta-\alpha \beta\right|+\left|\alpha \beta-\alpha \beta^{*}\right|}{(\alpha+\beta)\left(\alpha^{*}+\beta^{*}\right)}\right] \int_{0}^{T}\left(K_{1}\left|x^{*}(s)\right|+K_{1} \mid I^{\gamma} f_{2}\left(s, x^{*}(s)\right)\right)|+| f_{1}(s, 0,0) \mid\right) d s \\
& +\left(2 K_{1} T+2 K_{1} K_{2} \frac{T^{\gamma+1}}{\Gamma(\gamma+2)}\right)\left\|x-x^{*}\right\| \leq \frac{(2 \delta)\left|x_{0}\right|}{(\alpha+\beta)\left(\alpha^{*}+\beta^{*}\right)} \\
& +\left(\frac{2 \delta}{\left(\alpha^{*}+\beta^{*}\right)}\left(K_{1} r T+\left(K_{1} \int_{0}^{T} \mid I^{\gamma} f_{2}\left(s, x^{*}(s)\right)\right) \mid d s+m_{1} T\right)\right. \\
& +\left(2 K_{1} T+2 K_{1} K_{2} \frac{T^{\gamma+1}}{\Gamma(\gamma+2)}\right)\left\|x-x^{*}\right\| \leq \frac{(2 \delta)\left|x_{0}\right|}{(\alpha+\beta)\left(\alpha^{*}+\beta^{*}\right)} \\
& +\left(\frac{2 \delta}{\left(\alpha^{*}+\beta^{*}\right)}\right)\left(K_{1} r T+K_{1} \int_{0}^{T} I^{\gamma}\left(K_{2}|x(s)|+\left|f_{2}(s, 0)\right|\right) d s+m_{1} T\right) \\
& +\left(2 K_{1} T+2 K_{1} K_{2} \frac{T^{\alpha+1}}{\Gamma(\alpha+2)}\right)\left\|x-x^{*}\right\| \leq \frac{(2 \delta)\left|x_{0}\right|}{(\alpha+\beta)\left(\alpha^{*}+\beta^{*}\right)} \\
& +\left(\frac{2 \delta}{\left(\alpha^{*}+\beta^{*}\right)}\right)\left(K_{1} r T+\left(K_{1} K_{2} r+K_{1} m_{2}\right) \int_{0}^{t} \int_{0}^{s} \frac{(s-\tau)^{\gamma-1}}{\Gamma \gamma} d \tau d s+m_{1} T\right) \\
& +\left(2 K_{1} T+2 K_{1} K_{2} \frac{T^{\gamma+1}}{\Gamma(\gamma+2)}\right)\left\|x-x^{*}\right\| \leq \frac{(2 \delta)\left|x_{0}\right|}{(\alpha+\beta)\left(\alpha^{*}+\beta^{*}\right)} \\
& +\left(\frac{2 \delta}{(\alpha+\beta)\left(\alpha^{*}+\beta^{*}\right)}\right)\left(K_{1} r T+\left(K_{1} K_{2} r+K_{1} m_{2}\right) \frac{T^{\gamma+1}}{\Gamma(\gamma+2)}+m_{1} T\right) \\
& +\left(2 K_{1} T+2 K_{1} K_{2} \frac{T^{\gamma+1}}{\Gamma(\gamma+2)}\right)\left\|x-x^{*}\right\|,
\end{aligned}
$$


then

$$
\left\|x-x^{*}\right\| \leq \frac{\left.(2 \delta)\left|x_{0}\right|+2 \delta\left(K_{1} r T+\left(K_{1} K_{2} r+K_{1} m_{2}\right) \frac{T^{\gamma+1}}{\Gamma(\gamma+2)}\right)+m_{1} T\right)}{\left(\alpha^{*}+\beta^{*}\right)\left[1-\left(2 K_{1} T+2 K_{1} K_{2} \frac{T^{\gamma+1}}{\Gamma(\gamma+2)}\right)\right]} \leq \epsilon .
$$

\section{ANTI-PERIODIC NONLOCAL BOUNDARY VALUE PROBLEM.}

Consider the nonlocal boundary value problem of (2)-(3) with the anti-periodic nonlocal condition

$$
x(\tau)=-x(1-\tau) \quad \tau \in[0, T] .
$$

Corollary 1. If $\alpha=1, \beta=1$, and $\eta=1-\tau$, and $x_{0}=0$, in Theorem (1.2), then the anti-periodic boundary value problem.

$$
\begin{gathered}
\frac{d x}{d t}=f_{1}\left(t, x(t), I^{\gamma} f_{2}(t, x(t))\right), \quad \alpha \in(0,1) \quad t \in(0, T) \\
x(\tau)=-x(1-\tau) \quad \tau \in[0, T]
\end{gathered}
$$

has the at least one solution $x \in A C[0, T]$

$$
\begin{aligned}
x(t) & =-\int_{0}^{\tau} f_{1}\left(s, x(s), I^{\gamma} f_{2}(s, x(s))\right) d s-\int_{0}^{1-\tau} f_{1}\left(s, x(s), I^{\gamma} f_{2}(s, x(s))\right) d s . \\
& +\int_{0}^{t} f_{1}\left(s, x(s), I^{\gamma} f_{2}(s, x(s))\right) d s .
\end{aligned}
$$

Now, let $\tau=\frac{1}{2}$, then

$$
\begin{aligned}
& x(t)=-\int_{0}^{\frac{1}{2}} f_{1}\left(s, x(s), I^{\gamma} f_{2}(s, x(s))\right) d s-\int_{0}^{\frac{1}{2}} f_{1}\left(s, x(s), I^{\gamma} f_{2}(s, x(s))\right) d s . \\
& +\int_{0}^{t} f_{1}\left(s, x(s), I^{\alpha} f_{2}(s, x(s))\right) d s . \\
& =\int_{0}^{t} f_{1}\left(s, x(s), I^{\gamma} f_{2}(s, x(s))\right) d s-2 \int_{0}^{\frac{1}{2}} f_{1}\left(s, x(s), I^{\gamma} f_{2}(s, x(s))\right) d s .
\end{aligned}
$$

\section{CONCLUSIONS}

We proved here, under certain conditions, the existence of at least one continuous solution $x \in C[0, T]$ of the nonlocal two-point, with parameters $\left(\alpha, \beta\right.$ and $\left.x_{0}\right)$ boundary value problem (1)-(2). The maximal and minimal solutions of the problem (1)-(2) have been proved. The continuous dependence of the unique solution on the parameters $(\alpha$, $\beta$ and $x_{0}$ ) have been also proved. The anti-periodic boundary value problem have been considered as an application. 


\section{REFERENCES}

[1] Boucherif, A and Precup, R, On the nonlocal initial value problem for first order differential equations, Fixed Point Theory 4(2),(2003) 205-212.

[2] Curtain, R.F., Pritchard, A.J., 1977. Functional analysis in modern appliedmathematics. Academic press.

[3] EL-Sayed, A.M.A and Ibrahim, A.G, Multivalued fractional differential equations, Applied Mathematics and Computations, 68 (1995) 15-50.

[4] EL-Sayed, A.M.A and Ibrahim, A.G., Set-valued integral equation of fractional orders, Applied mathematics and Computatations 118(2001) 113-121.

[5] El-sayed, A.M.A and Bin-Taher, E. O.A, Nonlocal problem for a multi-term fractional order differential equation, Journal of Nath. Analysis, 29(2001) 1445-1451.

[6] A. M. A. El-Sayed, A. Elsonbaty, A. A. Elsadany and A. E. Matouk, Dynamical Analysis and Circuit Simulation of a New Fractional-Order Hyperchaotic System and Its Discretization, International Journal of Bifurcation and Chaos, 26( 13) (2016) 1650222.

[7] A. R. Elsonbaty and A. M. A. El-Sayed, Further nonlinear dynamical analysis of simple jerk system with multiple attractors, Nonlinear Dyn. 87(2), (2017) $1169 ? 1186$.

[8] Srivastava, H. M., El-Sayed, A. M. A and Gaafar, F. M, A Class of Nonlinear Boundary Value Problems for an Arbitrary Fractional-Order Differential Equation with the Riemann-Stieltjes Functional Integral and Infinite-Point Boundary Conditions, Symmetry 10, (2018), 508.

[9] El-Sayed, A. M. A and Ahmed, R.G, Infinite point and Riemann-Stieltjes integral conditions for an integro-differential equation, Nonlinear Analysis: Modelling and Control 24 (2019) 733-754.

[10] El-Sayed, A. M. A and Ahmed, R. G, Existence of solutions for a functional integro-differential equation with infinite point and integral conditions, International Journal of Applied and Computational Mathematics 5 (2019), 108.

[11] El-Sayed, A.M.A and Ahmed R.G, Solvability of a coupled system of functional integro-differential equations with infinite point and Riemann-Stieltjes integral conditions, Applied Mathematics and Computation 370 (2020) 124-918.

[12] El-Sayed, A.M.A and Al-Issa, S.M., Monotonic solutions for a quadratic integral equation of fractional order. AIMS Mathematics, 4(3)(2019) 821-830. 
[13] El-Sayed, A.M.A and Al-Issa, S.M., On a set-valued functional integral equation of Volterra-Stiltjes type. Journal of Mathematics and Computer Science, 21 (4) (2020) 273-285.

[14] Fierro, R. C. Martines.And, C. H. Morales, Caratheodory selections for multi-valued mappings, Nonlinear Analysis, 64 (2006) 1229-1235.

[15] Herzallah, M.A.E., El-Sayed, A.M.A and Baleanu, D., On the fractional-order diffusion-wave process, Romanian Reports of Physics 55, 3-4, (2010) 274-284.

[16] Podlubny, I., EL-Sayed, A.M.A., 1996. On two definitions of fractional calculus, Slovak Academy of science-institute of eyperimental phys." UEF-03-96 ISBN 80-7099-252.2 